\title{
Modeling a Smart Environment for Non-intrusive Analysis of Attention in the Workplace
}

\author{
Dalila Durães $^{1,3}$, Davide Carneiro ${ }^{2,3}$, Javier Bajo ${ }^{1}$, Paulo Novais ${ }^{2}$ \\ 1 Department of Artificial Intelligence, Technical University of Madrid, \\ Madrid, Spain \\ 2 Algoritmi Center, Minho University, Braga, Portugal \\ 3 CIICESI, ESTG, Polytechnic Institute of Porto, Felgueiras, Portugal
}

\begin{abstract}
Nowadays, the world is getting increasingly competitive and the quality and the amount of the work presented is one of the decisive factors when choosing an employee. It is no longer necessary to only perform but, to achieve a product with quality, on time, at the lowest possible cost and with the minimum resources. For this reason, the employee must have a high score of attention when performing a task and the factors that influence attention negatively must be reduced. This is true in many different domains, from the workplace to the classroom. In this paper we present a nonintrusive smart environment for monitoring people's attention when working in teams. The presented system provides real-time information about each individual, as well as information about the team. It can be very useful for team managers to identify potentially distracting events or individuals since when the attention of an individual is not at its best when performing the proposed task her/his performance will be negatively affected, with consequences for the individual as well as for the organization.
\end{abstract}

Keywords

Smart Environment, Attention Behavior, Non-intrusive, Distributed Computing.

\section{INTRODUCTION}

The rapid progress of wireless communication and sensing technologies enabled the development of smart learning environments, which are able to detect the environmental context as well as quantifying the attention of a worker in his workplace. For this reason, making intelligent learning systems has been the objective of many researchers in the field of computer science. 
In the field of computer science, a smart environment is a digitally augmented physical world where sensor-enabled and networked devices work continuously and collaboratively to make the lives of the inhabitants more comfortable. Indeed, significant advances in smart devices, wireless mobile communications, sensor networks, pervasive computing, machine learning, robotics, middleware and agent technologies, and human computer interfaces have made the dream of smart environments a reality. In this concept, the word "smart" means the ability to autonomously acquire and apply knowledge, and the word "environment" means our surroundings (Cook and Das, 2005).

With this technological evolution, job offers have changed, bringing along many significant and broad changes. Some of the most notorious ones can be pointed out by the emergence of indicators such as attentiveness which, in extreme cases, can compromise the life and wellbeing of the workers. In more moderate cases it will impair attention, general cognitive skills and productivity. In addition to these factors, many of these jobs are the so-called desk-jobs, in which people frequently sit for more than 8 hours (Liao and Drury, 2000).

Until now, the level of attention of a worker has been evaluated through his/her productivity: the more one produces, the better his/her attention at work. While the true nature of this relationship is yet to be thoroughly studied (properly contextualized in each work domain), there are other issues that need to be addressed. First, the worst aspect about this approach is that it only points out a potential decrease of attention after a productivity loss. This means that the "damage" is already done and that it is most likely too late for the worker to cope with whatever caused the attention loss. An approach that could point out, in advance, upcoming breaks in attention (e.g. through the observation of behavioral patterns) could allow active/preventive interventions rather than reactive ones (Carneiro, Novais, Andrade, Zeleznikow, and Neves, 2013).

Attention is a very complex process through which an individual continuously analyzes a spectrum of stimuli and, in a sufficiently short amount of time, choses one to focus on (Estes, 2014). In most of us, which can only focus on a reduced group of stimuli at a time, this implies ignoring other perceivable stimuli and information.

Research on attention involves nowadays many fields, including education, psychology, neuroscience, cognitive neuroscience and neuropsychology. For this reason, many different views and theories on attention can be found. One of the most frequent is the so-called Attention Economics, which treats human attention as a scarce commodity or resource, which we must use wisely to attain our goals (Davenport and Beck, 2013).

Individuals who have difficulties in focusing (their attention) can see the performance of other high-level cognitive processes, such as learning or decision making, negatively affected. In extreme cases, such as in 
Attention-Deficit/Hyperactivity Disorder (ADHD), this may have a significant negative impact in the development and function of the individual (ATTENTION-DEFICIT et al, 2011).

Although these aspects have always existed, in the last years we have seen an increase of distracting stimuli, which makes this topic a still more important one. Nowadays, we deal with constant notifications from our e-mail, our social networks, our messaging applications, advertisements and so on. We live immersed in beeps, vibrations, notifications and blinking icons, which constantly call for our attention and distract us (McBride, 2015). Even if we return immediately to our task, the fact that we had to consciously evaluate the stimuli to decide that it is not important at the moment already had a toll on our brain, making it spend resources (Davenport and Beck, 2013; Simola, Hyönä, and Kuisma, 2015).

This is especially worrying in young children, who nowadays have an easy access to computers, mobile phones and tablets, with their games and engaging applications. For them, it is so easy to get distracted by these stimuli, making learning less efficient and more frustrating, therefore negatively affecting their development (Gottlieb, 2012).

In this paper we present a distributed system for monitoring attention in teams (of people), in line with the vision of Smart Environments (Augusto, Callaghan, Cook, Kameas, Satoh, 2013). It is especially suited for people working with computers and it can be interesting for domains such as the workplace or the classroom. It constantly analyzes the behavior of the user while interacting with the computer and, together with knowledge about the task, is able to temporally classify attention.

This work may be very interesting for team managers to assess the level of attention of their teams, identifying potential distractions. Moreover, distraction often appears when the individual is fatigued, bored or not motivated. This tool can thus be an important indicator of the team, allowing the manager to act accordingly at an individual or group level. In the overall, this tool will support the implementation of better strategies for human resources management. 


\section{RELATED WORK}

Attention is one of the factors that influence the performance of a human being, when performing a task. If the attention of an individual is not at its best when performing the proposed task, his/her performance will be negatively affected, causing several problems.

In enterprise environments, if an employee shows lack of attention at work, that behavior can cause production or productivity issues. This has, as consequence, delays in delivery to costumers and in extreme situations it can cause monetary losses. In some situations, such as flight controllers or bus drivers, if the person is not $100 \%$ focused, an error can cause a tragic accident. In educational environments, attention is considered a fundamental factor in the evolution and success of the student. If the student is not concentrated and paying attention to what is being taught, he/she will not get the information that is being provided and consequently the academic results may be compromised.

\section{$2.1 \quad$ Attention}

Attention is a resource that allows the human being to be focused on a situation and to be able to ignore non-priority information. As it happens with performance, several factors such as stress, mental fatigue, anxiety, emotion, new environments, and human health can influence attention. Besides these factors, the development of technology has been a real problem which has increased the lack of attention. With the appearance of the smartphone which provides new and varied information in real time as well as new ways of communication, the individual's attention is easily captured and the task that was meant to be done is left out (Durães, Jiménez, Bajo, and Novais, 2016).

Generally, there is no universally accepted definition of attention because there is a diversity of subjects that are focused on it. In the past, only psychologists studied attention; however, these days attention is highly important for other fields like philosophy, chemistry, anatomy, and even computational science (Mancas, 2007).

The concept of attention may be defined as the transformation of a huge acquired unstructured data set into a smaller structured one, where the main information is preserved. In Computer Science, attention means that there is a filtering input space that selects the most important data in processing and this is a key mechanism of behavioral control for tasks, which is related to planning, decision making, and preventing new situations. However, there are limited computation capabilities (Mancas, 2007; Tamiz, Karami, Mehorabi, and Gidary, 2013). 
Attention implies the concentration of mental powers upon an object by close or carefully observing or listening, which is the ability or power to concentrate mentally.

\subsection{User Behavior}

Task execution using new technologies has been increasing in the last years. Therefore, one of the biggest concerns is to find ways to make this use as efficient and effective as possible. Nonetheless, task execution is not done similarly by all users. Each person has his/her own behavior. This behavior can be derived from several factors, such as biological characteristics of the user; characteristics of the task and environmental factors.

The recognition of these behaviors is already used in new technologies. For example, the recognition of users as a form of authentication on a device or software done by tracking behavioral biometrics, such as, fingerprints, face recognition or iris recognition (Benevenuto, et al., 2009).

According to Roman Yampolskiy and Venu Govindaraju (2008), these behavioral biometrics can be classified in five categories based on information type:

- Behavioral Biometric based on the analysis of text extracts or drawings produced by the user;

- Behavioral Biometric based on computer interaction;

- Device usage, such as mouse and keyboard. Devices that can capture the muscles' actions;

- Software interaction: strategy, and knowledge;

- Behavioral Biometric based on monitoring data coming from low level action in software, such as access log, storage activity, and calls systems;

- Behavioral Biometric based on data from motor skills;

One of the variables is attention. To recognize the lack of attention on user behavior, different ways of capture and classify attention were discovered. One of the first ways to try to quantify the attention level was a questionnaire. This questionnaire is presented to the user. After the user answers the questionnaire, the study's author analyses the answers and presents the conclusions. However, this approach is more qualitative and depends on the author's interpretation (Mancas, 2017).

The more quantitative approach is the one that use biometrics behavioral. It is an approach more focused on the perception stimuli. Eye-tracking is one of the most used techniques. Using this technology 
when performing a task on a computer, it is possible to know the screen area where the eyes are directed and, consequently, where is the focus of attention. So, it is possible to conclude if the user was with the visual attention directed to the screen area where the task is positioned (Mancas, 2017; Duc, 2008). This is, however a very intrusive methodology which raises privacy concerns, especially in the workplace.

Other approach is based on neural activity. The most used technique is EEG, which uses electrodes placed in the scalp that read the frequency of brain waves and, with the acquired data, analyse the brain activity during a task. In many studies, the most important component is MMN (mismatch negativity). This component is the indicator of a brain reaction to a pre-attention process. Other techniques used are: functional imaging, fMRI, MEG, functional imaging, and PET scan (Mancas, 2017).

Regarding techniques that measure attention, the first two techniques described are intrusive approaches. In the case of brain activity measures, it is necessary place some devices on the user's body to capture the data. This can interfere with the performance of the user and cause stress as it is intrusive. Regarding eye tracking, and although it does not require the use of devices placed on the body, the technique is an approach that has raises issues related to privacy and data protection (especially with underaged individuals), which makes it unsuitable for academic environments.

\subsection{Behavioral Biometrics}

Mouse and keyboard tracking are techniques also used to measure and classify attention. These techniques have already been used to measure other variables such as stress (Carneiro, 2015) and mental fatigue (Pimenta, 2013; Pimenta, 2015).

In the case of the stress study (Carneiro, 2015), the data was captured during an online exam of medical students. The captured data was related to mouse movement and keyboard usage. After the data were collected, the data were analyzed in two phases. In the first phase, it was used statistic methods and the first conclusions were obtained. This data was analyzed in two ways: to find common behavior in groups of students; individual data was analyzed for each student. In the second phase, it was used a machine learning method to model the students' response to a stress event. There were used two classifiers: one to classify the all students' data and other to classify data from each individual student. This study (Carneiro, 2015) concluded that stress affected the students' performance on an exam but also pointed out that some students can improve their performance even with stress. 
The mouse and keyboard tracking technique is a non-invasive approach because the data captured is compiled by a background software and the user does not have the perception that he/she is being monitored. This is an advantage over the two previous approaches as the user is not affected in any way by the collection of data.

\subsection{Smart Environments}

Important features of the already defined smart environments include a certain degree of autonomy, the capacity to adapt themselves to changing environments, and the communication with humans in a natural way (Cook, and Das, 2005). A smart environment is a tool in which technology is embedded, hidden in the background, sensitive, adaptive and responsive to the presence of people and objects. This system also preserves security and privacy while using information when needed and with an appropriate context (Weber, Rabaey, and Aarts, 2005). This is achieved by creating digital environments that are sensitive to people's needs and can respond to their requirements, anticipate behaviors and adjust the response accordingly (Costa, Julián, and Novais, 2017).

In this paper we propose an adaptive system that aims at supporting the monitoring work process. This system is adaptable, which means that it considers the worker's knowledge, background, interest, goals, targets and/or choices.

\section{A SMART ENVIRONMENT FOR ATTENTION MANAGEMENT}

To implement the proposed approach, a Smart Environment for attention management was developed. The key element in this environment is a data collection tool that registers all the events describing the user's interaction with the computer. These events support the generation of 14 interaction features, described in Section 3.1. These features, describing the interaction of each user with each device are processed, transformed and sent to a server, which continuously builds a user interaction profile, as described in Section 3.2.

Part of this framework was developed in previous work. The first version focused on the analysis of the individuals' interaction patterns with the computer, including features such as mouse velocity or acceleration, click duration, typing speed or rhythm, among others. For a complete list of features as well as the process of their acquisition and extraction, please see (Durães, Jiménez, Bajo, Novais, 2016). However, a limitation was also identified in this previous work. In fact, a user that 
opened a no work-related application and did not interact anymore with the computer until the end of the task had $0 \%$ of attention. On the other hand, if the user opens a work-related application and does not interact with the computer after that, the user's attention will be classified as $100 \%$ when he is most likely not even at the computer.

The present work adds a new feature to this previously existing framework, by providing a precise measure of attention based not on the key typing or mouse movement patterns but also on the actual application usage and switching patterns. It thus constitutes a much more precise and reliable mechanism for attention monitoring, while maintaining all the advantages of the existing system: nonintrusive, lightweight and transparent.

\subsection{Features Extraction}

While the user conscientiously interacts with the computer and takes his/her decisions and actions, a parallel and transparent process takes place in which the smart environment uses the information. The process of feature extraction starts with the acquisition of interaction events, which is carried out by a specifically developed application that is installed in each of the computers or smartphones. This application runs in the background and requires no interaction by the user. It is thus nonintrusive. The following events are acquired by the application and sent to the server for processing:

- MOV, timestamp, posX, posY. An event describing the movement of the mouse in a given time to coordinates (posX, posY) in the screen;

- MOUSE_DOWN, timestamp, [Left | Right], posX, posY. This event describes the first half of a click (when the mouse button is pressed down), in a given time. It also describes which of the buttons was pressed (left or right) and the position of the mouse in that moment;

- MOUSE_UP, timestamp, [Left | Right], posX, posY. An event similar to the previous one but describing the second part of the click, when the mouse button is released;

- MOUSE_WHEEL, timestamp, dif. This event describes a mouse wheel scroll of amount dif, in a given time;

- KEY_DOWN, timestamp, key. Identifies a given key from the keyboard being pressed down, at a given time;

- KEY_UP, timestamp, key. Describes the release of a given key from the keyboard, in a given time. 
The following example depicts a brief log that starts with some mouse movement (first two lines), contains a click with a little drag (lines 3-5) and ends with some more movement (last two lines).

MOV,635296941683402953,451,195

MOV,635296941684123025,451,197

MOUSE_DOWN,635296941684443057, Left ,451,199

MOV,635296941685273140,452,200

MOUSE_UP,635296941685283141, Left ,452,200

MOV,635296941685723185,452,203

MOV,635296941685803193,454,205

This subsection details the features that are extracted from the logs of interaction events. It is important to note that these features aim at quantifying user attention. Taking the mouse as example, its motion between two points is virtually never in a straight line as there is always some deviation. The larger this deviation, the less efficient the movement is. Of the following 13 considered features, 11 are extracted from the mouse and 2 from the keyboard:

Table 1. Data acquisition features.

\begin{tabular}{|c|c|c|}
\hline Symbol & Feature & Description \\
\hline \multicolumn{3}{|r|}{ Mouse Features } \\
\hline $\mathrm{mv}$ & Mouse Velocity & $\begin{array}{l}\text { The distance travelled by the mouse (in pixels) over the time } \\
\text { (in milliseconds). }\end{array}$ \\
\hline ma & Mouse Acceleration & $\begin{array}{l}\text { The velocity of the mouse (in pixels/milliseconds) over the } \\
\text { time (in milliseconds). }\end{array}$ \\
\hline $\mathrm{cd}$ & Click Duration & $\begin{array}{l}\text { The timespan between MOUSE_UP events, whenever this } \\
\text { timespan is inferior to } 200 \text { milliseconds. }\end{array}$ \\
\hline tbc & Time Between Clicks & $\begin{array}{l}\text { The timespan between two consecutive MOUSE_UP and } \\
\text { MOUSE_DOWN events, i.e., how long did it took the } \\
\text { individual to perform another click. }\end{array}$ \\
\hline $\mathrm{dbc}$ & $\begin{array}{l}\text { Distance Between } \\
\text { Click }\end{array}$ & $\begin{array}{l}\text { Represents the total distance travelled by the mouse between } \\
\text { two consecutive clicks, i.e., between each two consecutive } \\
\text { MOUSE_UP and MOUSE_DOWN events. }\end{array}$ \\
\hline ddc & $\begin{array}{l}\text { Duration Distance } \\
\text { Clicks }\end{array}$ & $\begin{array}{l}\text { The time between consecutive MOUSE_UP and } \\
\text { MOUSE_DOWN events. }\end{array}$ \\
\hline edbc & $\begin{array}{l}\text { Excess Distance } \\
\text { Between Clicks }\end{array}$ & $\begin{array}{l}\text { Represents the excess total distance travelled by the mouse } \\
\text { between two consecutive clicks, i.e., between each two } \\
\text { consecutive MOUSE_UP and MOUSE_DOWN events. }\end{array}$ \\
\hline aedbc & $\begin{array}{l}\text { Absolute Excess } \\
\text { Distance Between } \\
\text { Click }\end{array}$ & $\begin{array}{l}\text { This feature measures the average distance of the excess } \\
\text { total distance travelled by the mouse between two } \\
\text { consecutive clicks, i.e., between each two consecutive } \\
\text { MOUSE_UP and MOUSE_DOWN events. }\end{array}$ \\
\hline asdbc & $\begin{array}{c}\text { Absolute Sum } \\
\text { Distance Between } \\
\text { Clicks } \\
\end{array}$ & $\begin{array}{l}\text { This feature measures the average sum of distance that the } \\
\text { mouse travelled between each two consecutive MOUSE_UP } \\
\text { and MOUSE_DOWN events. }\end{array}$ \\
\hline dplbc & $\begin{array}{l}\text { Distance Point to Line } \\
\text { Between Clicks }\end{array}$ & $\begin{array}{l}\text { This feature will compute the distance between two } \\
\text { consecutive MOUSE_UP and } \\
\text { MOUSE_DOWN events. }\end{array}$ \\
\hline adpbc & $\begin{array}{l}\text { Absolute Distance } \\
\text { Point Between Clicks }\end{array}$ & $\begin{array}{l}\text { This feature will compute the average distance between two } \\
\text { consecutive MOUSE_UP and MOUSE_DOWN events. }\end{array}$ \\
\hline \multicolumn{3}{|r|}{ Keyboard Features } \\
\hline $\mathrm{kdt}$ & Key Down Time & $\begin{array}{l}\text { The timespan between two consecutive KEY_DOWN and } \\
\text { KEY_UP events. }\end{array}$ \\
\hline
\end{tabular}




\subsection{Real Time Analytics}

In the context of an organization, the gathering and analysis of metrics describing user behavior, and the providing of tools for visualization (particularly real-time analytics) enables better decision-making and data-driven actions that consider the state and well-being of each individual user. Such initiatives can nowadays be scaled to hundreds or thousands of users, through the use of Big Data tools and techniques, without compromising performance and availability. The features depicted in Section 3.1 describe the behavior of each individual while interacting with the computer. Each of these instances also contains a timestamp.

From the architecture of the developed environment described in Figure 1 it is possible to collect data that describe the interaction with both the mouse and the keyboard in the devices in which students work. These devices have software that generates raw data, which they store locally until it is synchronized with the web server in the cloud. This layer encodes each event with the corresponding necessary information (e.g. timestamp, coordinates, type of click, key pressed). These data are further processed, stored and then used to calculate the values of the behavioral biometrics. Mouse movements can also help to predict the state of mind of the user, as well as keyboard usage patterns.

After the raw data is stored in a data store engine, the analytic layer processes the data received (from the storage layer) in order to evaluate those data according to the metrics presented. Some data preparation tasks are also carried out in this phase, such as removing outliers (e.g. the backspace key being continuously pressed to delete a group of characters is not a regular key press). The system receives this information in real-time and calculates, at regular intervals, an estimation of the general level of performance and attention of each student.

In the classification profile layer the indicators are interpreted. Based on data from the attentiveness indicators and building the meta-data that will support decision-making, the system will classify the user profile. When the system has a sufficiently large dataset that allows making classifications with precision, it will classify the inputs received into different attention levels in real-time, creating each student learning profile. With these results it is possible to obtain a profile of the learning style. 


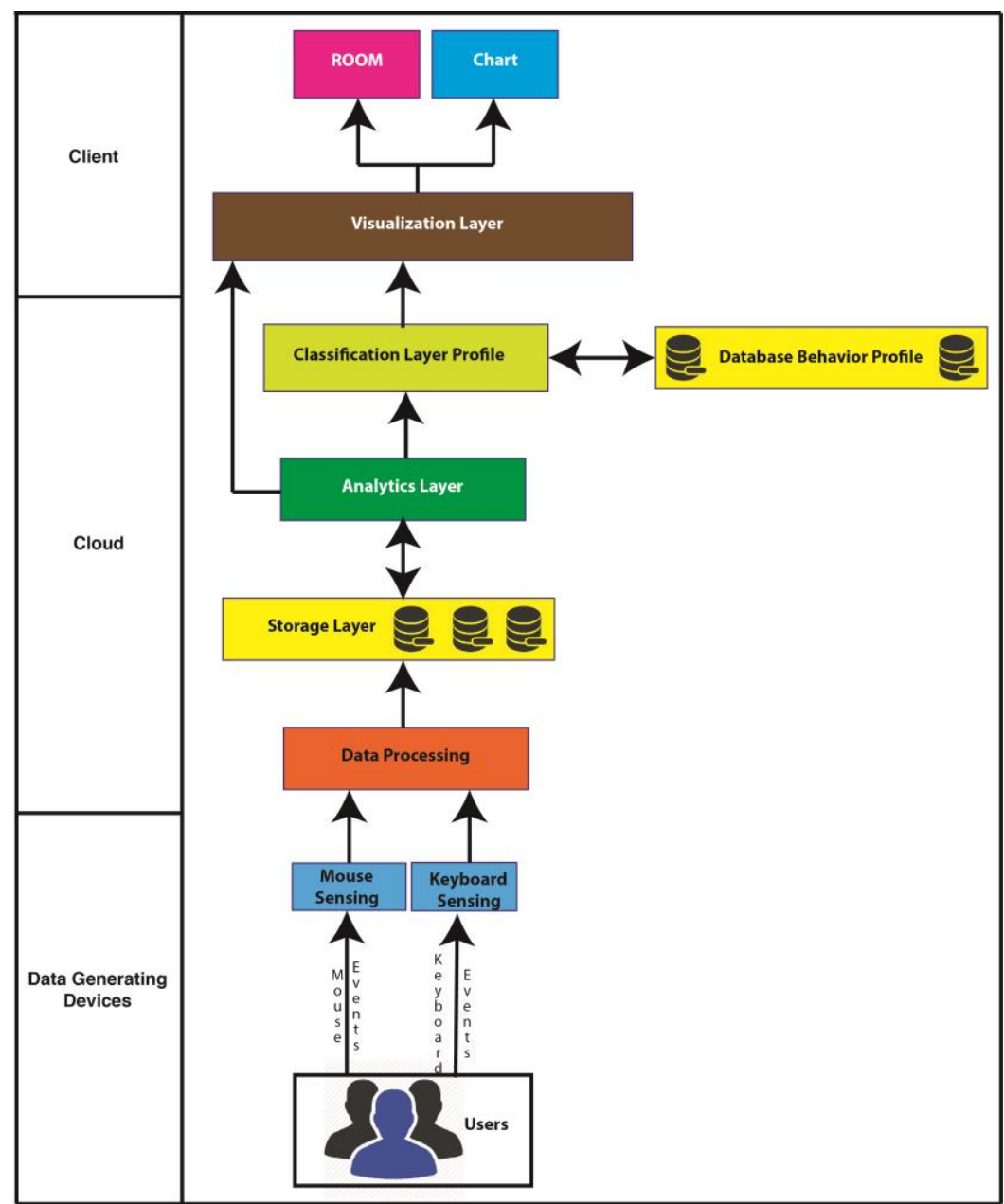

Fig. 1 Architecture of the smart system

Finally, the user attention information is displayed in the visualization layer, and it can be used to personalize instructions according to the specific user, enabling the administrator to act differently with different users as well as to act differently with the same user, according to his/her past and present level of attention. In the visualization layer it is possible to obtain some graphical modules that allow the display of information in an intuitive way to the user. This graphical module user interface is composed by a module that allows the creation of charts (CHART) and the layer that allows the creation of virtual teams (ROOM) so that the administrator may view intuitively the user's behavior.

\section{EXPERIMENTAL STUDY}

In our line of research we have been studying ways to assess attention level. In the present work, besides developing and presenting a smart environment for supporting the whole process, we also analyze the 
potential influence of external factors on attentiveness. In the early version of the system, the server classified the level of attention of the user based on the interaction patterns. With this work, the server now has access to a new type of raw data denoting that a given user switched to a specific application at a given timestamp. Since the server receives this data at regular intervals, it receives a list of triplets for each user. Thus, the new raw data received at regular intervals is as follows: (Id; Username; Timestamp; [(AppName; Timestamp)]). Id denotes the unique identifier of this group of data, Username uniquely identifies the user, Timestamp marks the end of the time interval for this group of data, and the last element is a list of pairs containing, in each element, the name of the app that the user switched to and the timestamp in which this happened.

This environment is designed to be used in the workplace by team managers to analyze the evolution of the attentiveness of the group during the day or during wider time periods. It will allow establishing each individual's optimum working cycle, improving productivity and well-being.

The present work adds a new feature to this previously existing framework, by providing for each task the level of interaction of the keyboard and of the mouse. It constitutes a more precise and reliable mechanism for attention monitoring, while maintaining all the advantages of the existing system: nonintrusive, lightweight, and transparent.

\subsection{Study Design}

As previously mentioned, a system with these characteristics may prove useful in very different domains, including organizational, academic or any environment in which people operate computers. To validate the proposed system, we have been using it for the past months in the Caldas das Taipas High School, located in northern Portugal. In the Portuguese academic context, this system gains increased relevance as current policies move towards the creation of larger classes, which make it increasingly difficult for the teacher to individually address to each student. In this section we show several tools supported by this system that, when at the disposal of the teachers may allow him/her to:

- Decide, in real-time, which students to focus on, according to their level of attention;

- Evaluate, a posteriori, which contents are more prone to generate distraction, providing a chance for improvement;

- Identify, in real-time, fluctuations in attention, improving decision-making concerning aspects such as when to make breaks or when to dismiss the class. 
In this work we compare the same class from a vocational course while performing an activity based on Microsoft Access in a lesson. The goal was to determine the level of attention when measured with the workrelated task and compared with mouse and keyboard interactions. With these results the smart environment can support future decision-making.

To validate this system we are following several cohorts of students during their academic activities. This data collection process will allow assessing the influence on attention of aspects such as: breaks, time of day, class contents, and class objectives, among others. For this purpose, a group of 14 (all girls) students were selected to participate in this experience. Their average age is 15.9 years old ( $\mathrm{SD}=1.5$ years). The experiment was applied in a lesson, where they had access to an individual computer and 100 minutes to complete the task. Students received, at the beginning of the lesson, all necessary data with the goals of the task. For this class, the lesson started in the afternoon and students received, at the beginning of the lesson, a document with the goals of the task, which in this case required the use of Microsoft Access and Adobe Acrobat Reader.

This application runs in the background, which makes the data acquisition process, a completely transparent one from the point of view of the student. It collects data from the students' interaction with the mouse and the keyboard, which act as sensors. The Mouse and Keyboard Sensing layers are responsible for capturing information describing the behavioral patterns of the students while interacting with the peripherals.

\subsection{Data Analysis}

In this sub-section we show the existence of different behaviors in the class. To quantify attentiveness the following methodology was followed. Asides from capturing the interaction of the user with the computer, the monitoring system also registers the applications with which the user is interacting. We analysed all the applications used by all users and labelled each of them as belonging to the task or not. We then quantified the amount of time that each user spent interacting with applications related to the task versus other applications. In this sense, the data were analyzed in two different ways: first, a general analysis was carried out in which statistics methods are used to obtain preliminary conclusions; secondly an individual analysis was done to compare the different moments.

Algorithm 1 presents the code to obtain the list of pairs and computes the time during which each window was active in order to obtain the time that the user spent on each task. However, there are often cases in which the user does not change applications for a large amount of time. In these cases, which are represented by a pair with an empty AppName, 
the time is added to the last known AppName (since this means that the user is still interacting with it).

Algorithm 1: Creating triplets with the intervals and timestamp of each application.

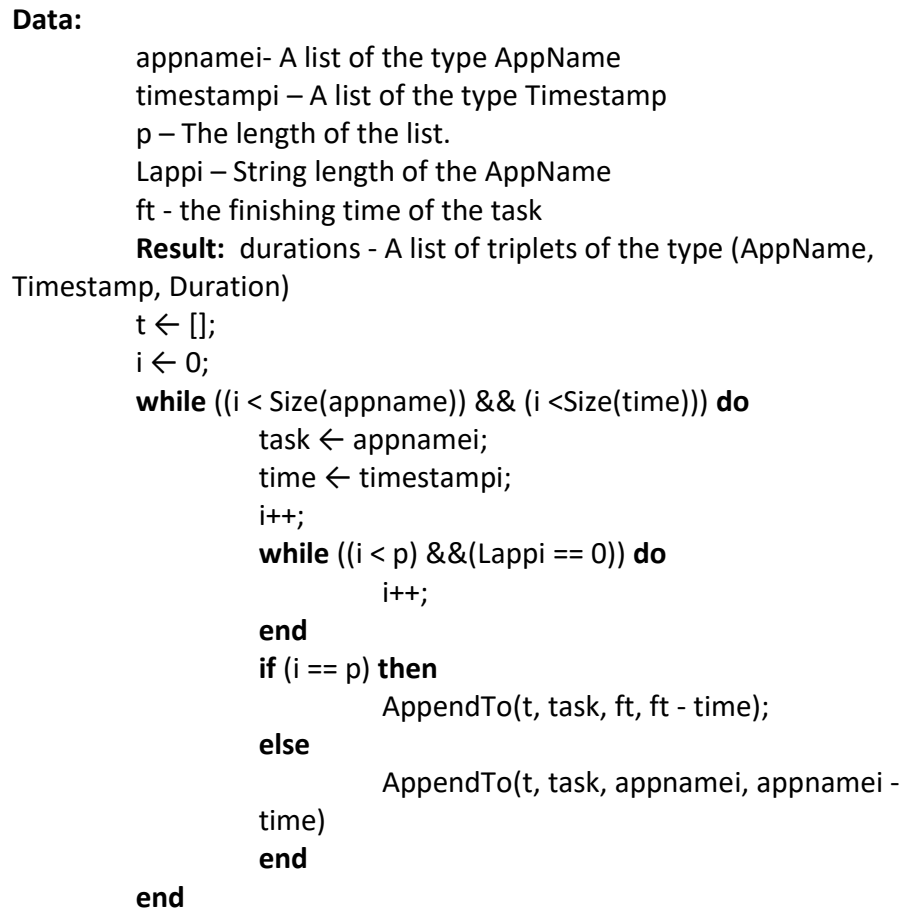

To identify the work-related applications, we analyzed all different applications used by all users and labeled each one as belonging to the task or not. We then quantified the amount of time that each student spent interacting with applications related to the task versus other applications. For that, it was necessary to compute the level of attention of the user as detailed in Algorithm 2.

Algorithm 2: Creating triplets at regular intervals with the timestamp the quantification of work-related.

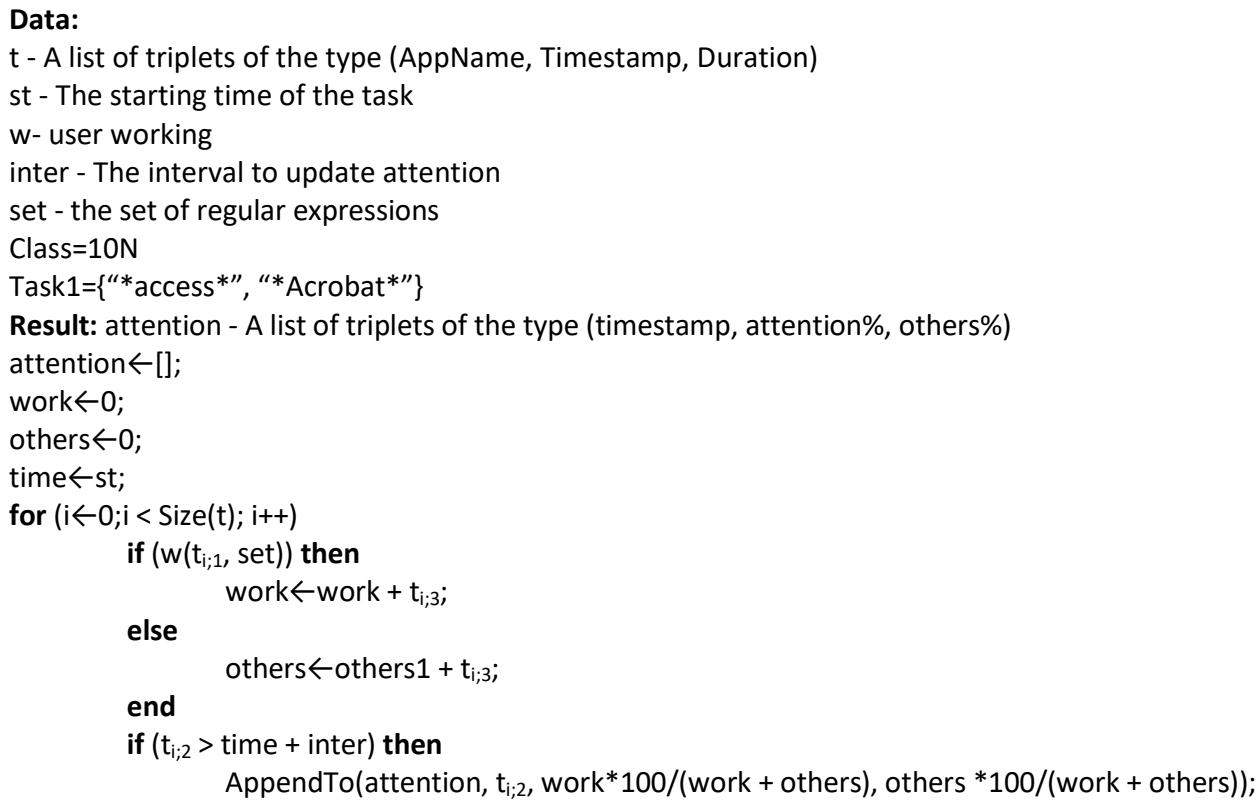




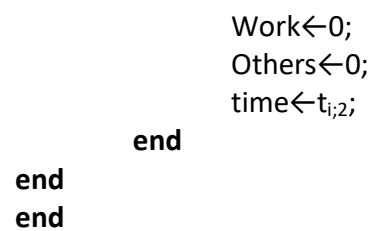

To do this we measured the amount of time, in each interval, that the user spent interacting with work-related applications. The algorithm thus needs knowledge about the domain in order to classify each application as belonging or not to the set of work-related applications. This knowledge is provided by the teacher and is encoded in the form of regular expressions. The teacher uses a graphical interface to set up rules such as "starts_with Access" or "contains Access", which are then translated to regular expressions that are used by the algorithm to determine which applications are work-related and which are not.

Whenever an application that does not match any of the known rules for the specific domain is found, the application name is saved so that the teacher can later decide if a new rule should or should not be created for it. By default, applications that are not considered work-related are marked as "others" and count negatively towards the quantification of attention.

\section{RESULTS}

In this section, and as mentioned in subsection 2.4 , we focus on the new feature that was added to the previously existing framework. We present an example with all the necessary steps to calculate the level of attention of the users. Thence, we briefly analyze the data collected for the same cohort of students $(10 \mathrm{~N})$. Firstly, it is necessary to know the interaction with the mouse and with the keyboard that each task will have. In some tasks the interaction with the mouse will be higher while in others that will happen with the keyboard, and finally in others, it will practically be the same level of interaction. However, it is difficult to know, a priori, what the exact percentage of interaction of the mouse or the keyboard will be. To know these values, we first count the number of times that each key is pressed and the number of times that the mouse is clicked down for each user. In Figure 2 we presented the results of the total number of keys pressed for the keyboard and the total number of mouse clicks for each user in the overall of the lesson. In this lesson, as mentioned in Section 4.1, the tasks defined were exercises in Microsoft Access. 


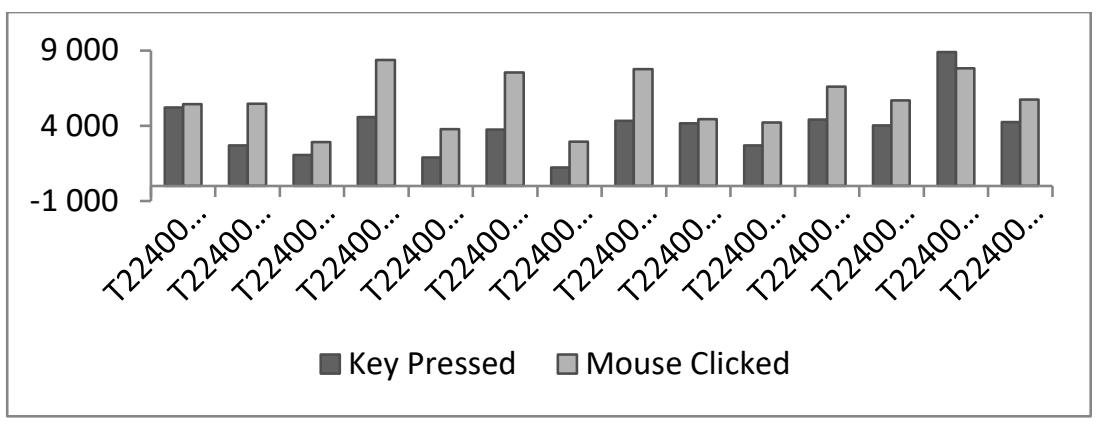

Fig. 2 Global results of the mouse down and key down for each user in the lesson.

Secondly, we calculated the percentage of the interaction of the mouse, which is the average number of times that the mouse was pressed in the class taking into account the total of interactions with the mouse and the keyboard of the class. Similarly, we calculated the percentage of the interaction of the keyboard, which is the average number of times that the keys were pressed in the class taking into account the total of interactions with the mouse and the keyboard of the class. In Table 2 it's presented the average of the interactions of the mouse and keyboard in the class and the global average percentage of the interaction of the mouse (59\%) and keyboard (41\%) of the class in this lesson.

Table 2. The average interaction of the mouse and the keyboard of the class for this lesson.

\begin{tabular}{cccccc}
\hline & Key Pressed & $\begin{array}{c}\text { Interaction } \\
\text { Key pressed }\end{array}$ & $\begin{array}{c}\text { Mouse } \\
\text { Clicked }\end{array}$ & $\begin{array}{c}\text { Interaction } \\
\text { Mouse Clicked }\end{array}$ & $\begin{array}{c}\text { Total } \\
\text { Interacti }\end{array}$ \\
\hline $\begin{array}{c}\text { Average } \\
\text { Users }\end{array}$ & 3.875 & $41 \%$ & 5626,64 & $59 \%$ & 9.501 \\
\hline
\end{tabular}

On the order hand, we obtained the amount of time that each student spent at the computer (Task Duration) as well as the amount (and percentage) of time that each student devoted to work and to other activities in the lesson as explained in the algoritm 1 of the preview section. Table 3 allows the teacher to analyze this result.

Table 3. The amount of time that each student spent interacting with the computer and the amount of actual work versus the amount of time spent interacting with other applications.

\begin{tabular}{rrrr}
\hline Student & Task Duration & \multicolumn{1}{c}{ Work \% } & \multicolumn{1}{c}{ Others \% } \\
\hline $\mathrm{T} 2240001$ & $85.0084 \mathrm{~min}$ & $0,0 \%$ & $100,0 \%$ \\
\hline $\mathrm{T} 2240002$ & $76.0154 \mathrm{~min}$ & $78.7 \%$ & $21.3 \%$ \\
\hline $\mathrm{T} 2240003$ & $90.0093 \mathrm{~min}$ & $69,7 \%$ & $30,3 \%$ \\
\hline $\mathrm{T} 2240004$ & $90.0065 \mathrm{~min}$ & $56,8 \%$ & $43,2 \%$ \\
\hline $\mathrm{T} 2240005$ & $90.0103 \mathrm{~min}$ & $57,0 \%$ & $43,0 \%$ \\
\hline $\mathrm{T} 2240006$ & $90.0083 \mathrm{~min}$ & $60,6 \%$ & $39,4 \%$ \\
\hline $\mathrm{T} 2240007$ & $95.0146 \mathrm{~min}$ & $61,8 \%$ & $38,2 \%$ \\
\hline $\mathrm{T} 2240008$ & $70.0049 \mathrm{~min}$ & $83,7 \%$ & $16,3 \%$ \\
\hline $\mathrm{T} 2240009$ & $70.0069 \mathrm{~min}$ & $88,6 \%$ & $11,4 \%$ \\
\hline
\end{tabular}




\begin{tabular}{llll}
\hline $\mathrm{T} 2240011$ & $75.0066 \mathrm{~min}$ & $83,7 \%$ & $16,3 \%$ \\
\hline $\mathrm{T} 2240012$ & $77.0067 \mathrm{~min}$ & $85.6 \%$ & $14.4 \%$ \\
\hline $\mathrm{T} 2240013$ & $70.0048 \mathrm{~min}$ & $75,5 \%$ & $24,5 \%$ \\
\hline $\mathrm{T} 2240014$ & $75.006 \mathrm{~min}$ & $70,2 \%$ & $29,8 \%$ \\
\hline
\end{tabular}

However, the time that the student spent in the task-related doesn't indicate the level of attention in some cases, because in some situations the user might have opened the task-related and during that time didn't interact with the computer. In this case, if the level of attention was measured only by the task-related, the student has $100 \%$ of attention and in reality his attention level should be $0 \%$. For these situations, it is necessary to analyze the amount of interaction with the mouse and the keyboard and cross these data.

The next step is to calculate the percentage of usage of the mouse for each user. The formula to calculate this value is:

$$
\begin{aligned}
& \text { Interaction_Mouse }=\text { Mouse_Down Count } / \text { MAX } \\
& \text { Mouse_Down Count }
\end{aligned}
$$

Where:

Mouse_Down Count: is the number of the times that the mouse was pressed by a user;

MAX Mouse_Down Count: is the higher score of usage of the mouse down in the class.

Similarly, for calculating the percentage of usage of the keyboard for each user, the formula for this value is:

$$
\text { Interaction_Keyboard = Key_Down Count / MAX Key_Down }
$$

Count

(2)

Where:

Key_Down Count: is the number of the time that one key was pressed by a user;

MAX Key_Down Count: is the higher score of usage of the Keyboard in the class.

Based on the formulas (1) and (2), the results presented in Table 4 were obtained, which contain the results for all students in this lesson.

Table 4. Percentage of use of the mouse and of the keyboard for each user in the lesson.

\begin{tabular}{ccccc}
\hline & Key Pressed & $\begin{array}{c}\text { Interaction Key } \\
\text { pressed }\end{array}$ & $\begin{array}{c}\text { Mouse } \\
\text { Clicked }\end{array}$ & $\begin{array}{c}\text { Interaction Mouse } \\
\text { Clicked }\end{array}$ \\
\hline $\mathrm{T} 2240001$ & 5.222 & 0,30 & 5433,772291 & 0,31 \\
\hline $\mathrm{T} 2240002$ & 2.694 & 0,16 & 5476,568369 & 0,32 \\
\hline $\mathrm{T} 2240003$ & 2.059 & 0,12 & 2929,010092 & 0,17 \\
\hline
\end{tabular}




\begin{tabular}{lllll}
\hline T2240004 & 4.588 & 0,27 & 8367,811556 & 0,48 \\
\hline T2240005 & 1.887 & 0,11 & 3786,672777 & 0,22 \\
\hline T2240006 & 3.758 & 0,22 & 7551,871827 & 0,44 \\
\hline T2240007 & 1.229 & 0,07 & 2947,859522 & 0,17 \\
\hline T2240008 & 4.328 & 0,25 & 7756,346945 & 0,45 \\
\hline T2240009 & 4.169 & 0,24 & 4430,711201 & 0,26 \\
\hline T2240010 & 2.698 & 0,16 & 4234,255905 & 0,25 \\
\hline T2240011 & 4.406 & 0,26 & 6615,953595 & 0,38 \\
\hline T2240012 & 4.037 & 0,23 & 5689,401539 & 0,33 \\
\hline T2240013 & 8.910 & 0,52 & 7817,398433 & 0,45 \\
\hline T2240014 & 4.259 & 0,25 & 5735,391528 & 0,33 \\
\hline
\end{tabular}

Finally, in order to obtain the level of attention it is necessary to combine the results of the interaction of the behavior biometrics of the Table 4 with the results showed in the time that each student spent in the task-related, presented previously in Table 3. This level of attention is the relative attention of each student, as this level of attention is compared with the other user of the class. The formula that calculated these values is:

Relative Attention $=($ Perc. Mouse $*$ Work-related $)+($ Perc. Keyboard * Work-related)

Where:

Perc. Mouse is the percentage of utilization of the Mouse for a user;

Perc. Keyboard is the percentage of usage of the Keyboard for a user;

Work-related is the time of a user spent in the task defined for the lesson.

Formula (3) provides the results for each user, presented in Table 5.

Table 5. Relative attention of each user in the lesson

\begin{tabular}{ccccc}
\hline User & $\begin{array}{c}\text { Relative } \\
\text { MouseDown }\end{array}$ & $\begin{array}{c}\text { Relative } \\
\text { KeyDown }\end{array}$ & Work-related & Relative Attention \\
\hline $\mathrm{T} 2240001$ & $31,45 \%$ & $30,22 \%$ & $0,00 \%$ & $0,00 \%$ \\
\hline $\mathrm{T} 2240002$ & $31,70 \%$ & $15,59 \%$ & $78,70 \%$ & $37,22 \%$ \\
\hline $\mathrm{T} 2240003$ & $16,95 \%$ & $11,92 \%$ & $69,73 \%$ & $20,13 \%$ \\
\hline $\mathrm{T} 2240004$ & $48,43 \%$ & $26,55 \%$ & $56,77 \%$ & $42,56 \%$ \\
\hline $\mathrm{T} 2240005$ & $21,92 \%$ & $10,92 \%$ & $56,95 \%$ & $18,70 \%$ \\
\hline $\mathrm{T} 2240006$ & $43,71 \%$ & $21,75 \%$ & $60,60 \%$ & $39,67 \%$ \\
\hline $\mathrm{T} 2240007$ & $17,06 \%$ & $7,11 \%$ & $61,81 \%$ & $14,94 \%$ \\
\hline $\mathrm{T} 2240008$ & $44,89 \%$ & $25,05 \%$ & $83,73 \%$ & $58,56 \%$ \\
\hline $\mathrm{T} 2240009$ & $25,64 \%$ & $24,13 \%$ & $88,58 \%$ & $44,09 \%$ \\
\hline $\mathrm{T} 2240010$ & $24,51 \%$ & $15,61 \%$ & $61,77 \%$ & $24,78 \%$ \\
\hline $\mathrm{T} 2240011$ & $38,29 \%$ & $25,50 \%$ & $83,72 \%$ & $53,41 \%$ \\
\hline $\mathrm{T} 2240012$ & $32,93 \%$ & $23,36 \%$ & $85,60 \%$ & $48,19 \%$ \\
\hline
\end{tabular}




\begin{tabular}{lllll}
\hline $\mathrm{T} 2240013$ & $45,25 \%$ & $51,57 \%$ & $75,52 \%$ & $73,11 \%$ \\
\hline $\mathrm{T} 2240014$ & $33,20 \%$ & $24,65 \%$ & $70,21 \%$ & $40,62 \%$ \\
\hline
\end{tabular}

Figure 3 presents the graphically result between percentage of time interacting with work-related applications and relative attention. It can be observed that the relative attention decreases in general, because the system takes in account the interaction of the user with mouse and keyboard. That is, someone who spent $100 \%$ of the time using the application that was supposed to but was interacting only $50 \%$ of the time, would have a score of attention of $50 \%$.

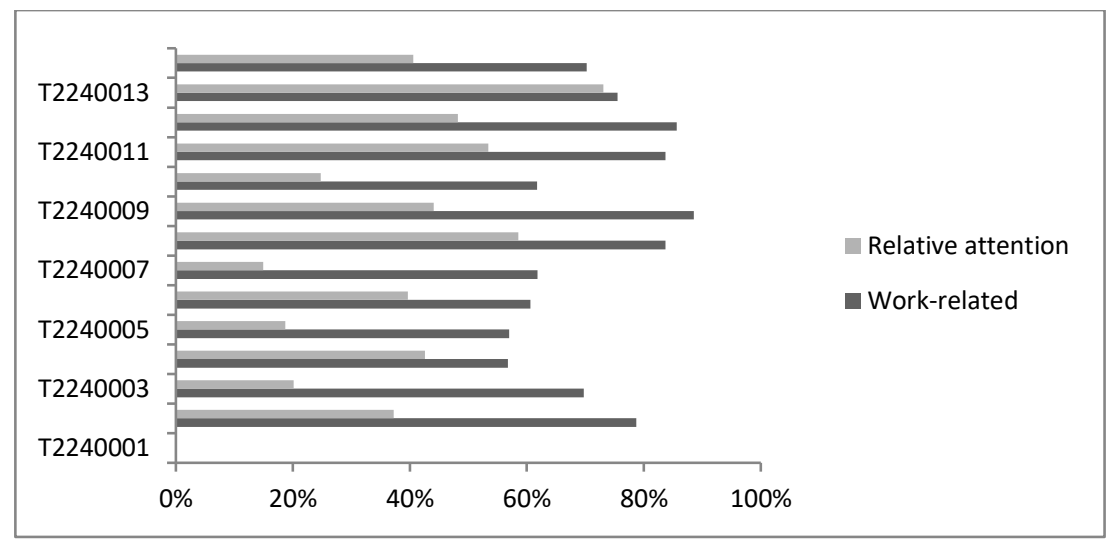

Fig. 3 Comparing work-related interaction with relative attention for each student.

\section{DISCUSSION AND CONCLUSIONS}

The task data from the user's interaction with the computer is the most crucial information because it derives most part of the attention level. To obtain the task results, the task rules received in the request are used to get a perception of how much time the user has spent on the applications related with the task rules.

As far as the mouse and keyboard results are concerned, their role is present on how the user interaction is occurring and it helps the manager/teacher to understand if any user, who has the application active, is really working on it or not. To do that, we have used the two features one from the keyboard (key down time) and other from the mouse (time between clicks).

Concerning attention, it is an important theme because it is one of the factors that most influences a person's performance while performing a task. Therefore, it is a much-studied theme by several areas, such as psychology, neuroscience and computer science. Understanding how attention varies and in which situations the attention varies for each person, it is possible to act in the right moment and right time, to bring the user's attention level to the ideal value. 
In order to obtain better results it is necessary to supervised users over a long period of time in order to create a more reliable data. Using this, the manager may take different measures depending on the profile of the group and/or profile of each user. The Classification layer has access to the current and historical state of the group from a global perspective, but can also refer to each user individually. After the classification, the enhanced user behavior profile is updated in the Database Behavior Profile.

A framework was proposed to address these issues, especially to monitoring user. Narrowing the scope of the study, a process to detect attentiveness was proposed, through the use of a developed log tool. With this smart environment it is possible to detect potentially negative factors dynamically and non-intrusively, making it possible to foresee negative situations, allowing to take actions to mitigate them. This may, in turn, minimize issues such as stress and anxiety, which can negatively influence the user' results and are closely related to the occurrence of conflicts. The work developed so far resulted in a useful system for the team manager, who can monitor, in real-time, the level of attention of users.

The door is thus open to intelligent platforms that allow to analyze user's profiles, taking into account their individual characteristics, and to propose new strategies and actions. By providing managers with access to this information, we allow them to better manage their interactions with the users, namely by pointing out the most problematic cases of inattention in real-time.

\section{ACKNOWLEDGEMENTS}

This work has been supported by COMPETE: POCI-01-0145-FEDER007043 and FCT - Fundação para a Ciência e Tecnologia within the Project Scope: UID/CEC/00319/2013.

\section{REFERENCES}

ATTENTION-DEFICIT, S.O., et al.: Adhd: Clinical Practice Guideline for the Diagnosis, Evaluation, and Treatment of Attention-Deficit/Hyperactivity Disorder in Children and Adolescents, Pediatrics, peds-2011.

Augusto, J.C., Callaghan, V., Cook, D., Kameas, A., Satoh, I.: Intelligent environments: a Manifesto. Human-Centric Computing and Information Sciences 3(1), 1 - 18, 2013.

Benevenuto, F., et al.: Characterizing User Behavior in Online Social Networks, IMC '09 Proceedings of the 9th ACM SIGCOMM Conference on Internet Measurement, 49-62, 2009. 
Carneiro, D., Novais, P., Andrade, F., Zeleznikow, J., Neves, J.: Using Case-Based Rea-soning and Principled Negotiation to Provide Decision Support for Dispute Resolution, Knowl. Inf. Syst. 36 (3), 789-826, 2013.

Carneiro, D., et al.: Using Mouse Dynamics to Assess Stress During Online Exams, pp. 345-356, 2015.

Cook, D. J., Das, S. K.: Smart Environments: Technology, Protocols, and Applications, Wiley, 2005.

Costa, A., Julián, V., Novais, P.: Advances and Trends for the Development of Ambient-Assisted Living Platforms, Expert System, 2017.

Duc, A. H., Bays, P., Husain, M.: Eye Movements as a Probe of Attention, Chapter 5.5, [book auth.] Christopher Kennard and R. John Leigh. Progress in Brain Research, pp. 403-411, 2008.

Durães, D., Jiménez, A., Bajo, J., Novais, P.: Monitoring Level Attention Approach in Learning Activities, Advances in Intelligent System and Computing, 478, 33-40, 2016.

Davenport, T.H., Beck, J.C.: The Attention Economy: Understanding the New Currency of Business, Harvard Business Press, 2013.

Estes, W.K.: Handbook of Learning and Cognitive Processes (Volume 4): Attention and Memory, Psychology Press, 2014.

Gottlieb, J.: Attention, Learning, and the Value of Information, Neuron 76(2), 281 - 295, 2012.

Liao, M.-H., Drury, C.: Posture, Discomfort and Performance in a vdt task, Ergonomics 43 (3), 345-35, 2000.

Mancas, M.: How to Measure Attention? News and insights from EAI community - Cited: 7 December 2017. http://blog.eai.eu/how-to-measureattention/.

Mancas, M.: Computational Attention - Toward Attentive Computers, In: Press Universi-taire de Louvain, 2007.

McBride, D.L.: Distraction of Clinicians by Smartphones in Hospitals: a Concept Analysis, Journal of advanced nursing 71(9), 2020 - 2030, 2015.

Pimenta, A., et al.: Monitoring Mental Fatigue through the Analysis of Keyboard and Mouse Interaction Patterns. [book auth.] Jeng-Shyang Pan, et al. Hybrid Artificial Intelligent Systems, pp. 222-231, 2013.

Pimenta, A., et al.: Detection of Distraction and Fatigue in Groups through the Analysis of International Patterns with Computers. [book auth.] David Camacho, et al. Intelligent Distributed Computing VII. s.1. : Springer International Publishing, pp. 29-39, 2015.

Simola, J., Hyönä, J., Kuisma, J.: Perception of Visual Advertising in Different Media: From Attention to Distraction, Persuasion, Preference and Memory, Frontiers Media SA, 2015.

Tamiz, M., Karami, M., Mehorabi, I., Gidary, S. S.: A Novel Attention Control Modeling Method for Sursor Selection Based on Fuzzy Neural 
Network Learning, First RSI/ISM In-ternational Conference on Robotics and Mechatronics (ICRoM), 2013.

Yampolskiy, R. V., Govindaraju, V.: Behavioural Biometrics: a Survey and Classification, International Journal of Biometrics, 2008.

Weber, W., Rabaey, J. M., Aarts, E.: Ambient Intelligence, Springer, 1-2, 2005. 